\title{
Searching for High Baryon Density at the AGS with ARC
}

\author{
S.H. Kahana, T.J. Schlagel and Y. Pang \\ Physics Department \\ Brookhaven National Laboratory \\ Upton, New York 11973
}

\begin{abstract}
A relativistic cascade ARC is used to analyse heavy ion experiments at the AGS. In particular predictions from ARC for $\mathrm{Au}$ on $\mathrm{Au}$ at 11.6 $\mathrm{GeV} / \mathrm{c}$ have proved to be remarkably accurate. Going to lower energies and inserting a phenomenological equation of state into the cascade should lead to information about the interesting region of high baryon density.
\end{abstract}

Talk presented by S.H.K. at Quark Matter ' 93

Tenth International Conference on Ultra-Relativistic

Nucleus-Nucleus Collisions

Borlänge, Sweden

June $20-24, .1993$

This manuscript has been authored under contract number DE-AC02-76CH00016 with the U.S. Department of Energy. Accordingly, the U.S. Government retains a non-exclusive, royalty-free license to publish or reproduce the published form of this contribution, or allow others to do so, for U.S. Government purposes.

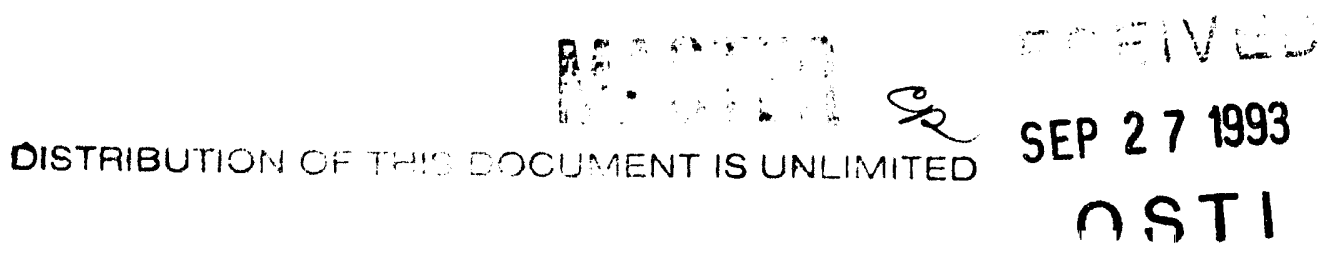




\section{DISCLAIMER}

This report was prepared as an account of work sponsored by an agency of the United States Government. Neither the United States Government nor any agency thereof, nor any of their employees, makes any warranty, express or implied, or assumes any legal liability or responsibility for the accuracy, completeness, or usefulness of any information, apparatus, product, or process disclosed, or represents that its use would not infringe privately owned rights. Reference herein to any specific commercial product, process, or service by trade name, trademark. manufacturer, or otherwise does not necessarily constitute or imply its endorsement, recommendation, or favoring by the United States Government or any agency thereof. The views and opinions of authors expressed herein do not necessarily state or reflect those of the United States Government or any agency thereof. 
Searching for High Baryon Density at the AGS with ARC

S. H. Kahana, T. J. Schlagel, and Y. Pang

Department of Physics, Brookhaven National Labratory, Upton, NY 11973, USA

A relativistic cascade ARC is used to analyse heavy ion experiments at the AGS. In particular predictions from $\mathrm{ARC}$ for $\mathrm{Au}$ on $\mathrm{Au}$ at $11.6 \mathrm{GeV} / \mathrm{c}$ have proved to be remarkably accurate. Going to lower energies and inserting a phenomenological equation of state into the cascade should lead to information about the interesting region of high baryon density.

\section{INTRODUCTION}

Principally, I wish to demonstrate in this talk the predictability inherent in a properly constructed cascade for relativistic heavy ion reactions, in particular for $\mathrm{Au}+\mathrm{Au}$ collisions at the $\mathrm{AGS}^{1,2}$. One important byproduct of this result is the reliable information about the baryon and energy densities one obtains in these collisions of truly massive nuclei. The $\mathrm{Au}+\mathrm{Au}$ environment at low energy may not yield direct knowledge of the meson-dominated plasma expected at central rapidities at RHIC, but certainly may provide an excellent playing field for consideration of the very interesting high baryon density region of phase space. For a more complete description of the approach we take in ARC I refer you to my HIPAGS ${ }^{1}$ talk and the references therein. Here I will concentrate on the experiment E866 involving a $11.6 \mathrm{GeV} / \mathrm{A}$ gold beam. ARC predictions $s^{1,3}$ for these gold on gold collisions have proven to be remarkably accurate.

$\mathrm{ARC}^{1,2}$ has now met most challenges offered by data collected at the AGS in the experiments $^{4}$ E802, E814, E810 and most recently E866 ${ }^{3}$. ARC gives an excellent description of E802 data covering ion reactions for initial beam momenta up to 14.6 $\mathrm{GeV} / \mathrm{c}$ and for observed particles up to midrapidity. ARC is equally good for forward protons seen by E814 ${ }^{4}$ in $S i+P b$ for rapidities up to 3.6, thus providing a single consistent picture of the entire rapidity range examined at the AGS. To complete this panorama, ARC has been used also to analyze the production of antiparticles and of light clusters $(d, t, h)$ in $p+A$ and $B+A$ collisions. To those seeking deviations from cascade predictions: it is highly unlikely anything will show up in light-ion (e.g. Si) induced reactions, where densities are lower and last for shorter times.

\section{PHYSICS IN ARC}

A crucial element in the development of this cascade was the recognition that the dynamics of rescattering is dominated by resonances produced in hadron-hadron collisions. At the AGS this is especially true for baryon-resonances, although also important for mesons. Such rescatterings most frequently involve resonance-nucleon interactions but even resonance-resonance collisions occur and must be treated. Two features are of prime importance. First the low lying resonances have appreciable times which then 
take precedence over particle formation times, at AGS energies. Secondly, leading baryon resonances possess higher momentum when colliding than would the corresponding hadron. For example, $\Delta$ and $N^{*}$, which play a crucial rule at the AGS, have momenta $k_{\Delta} \sim k_{N}+k_{\pi}>k_{N}$. This by itself explains two perplexing features of the AGS data: 1) The broadening in proton $m_{t}$ spectra i.e. the large proton temperatures. 2) The increase in $\mathrm{K}^{+}$production. These follow because the resonance interactions are: (1) at higher energies and therefore more inelastic, and (2) also are higher above the strangeness thresholds.

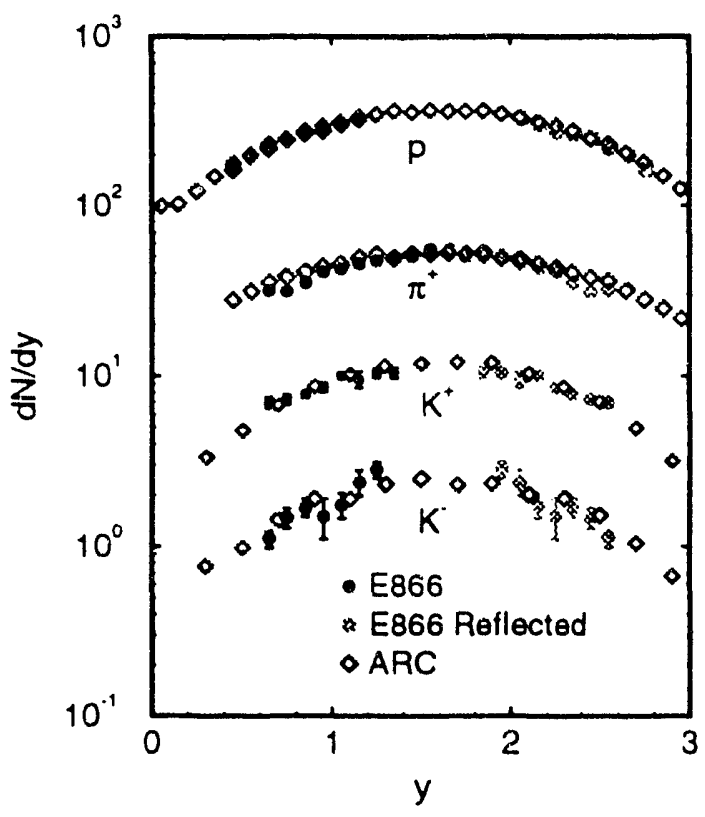

Figure 1. Rapidity distributions for protons (x5), pions and kaons, compared to E866.

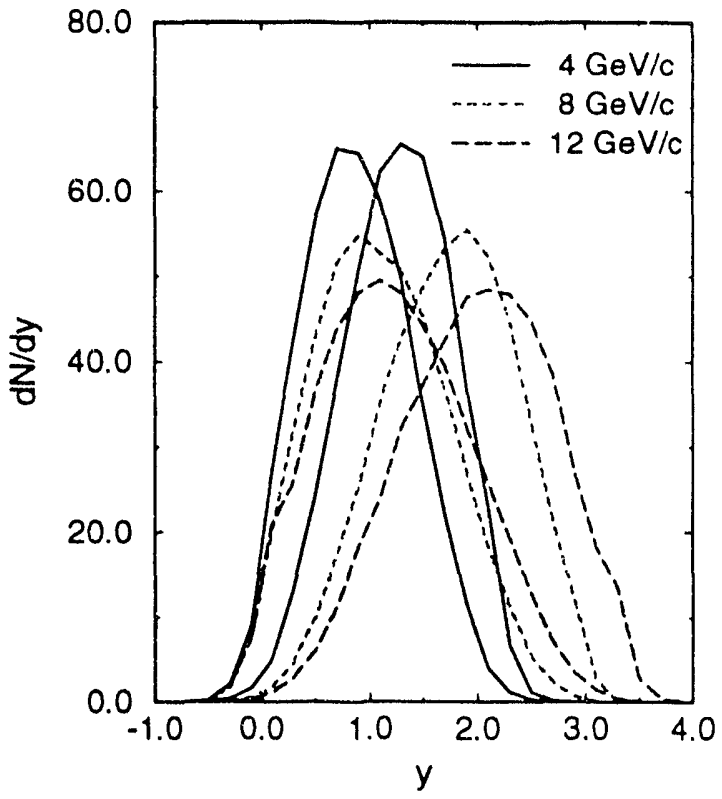

Figure 2. Target and projectile protons rapidity spectra separately for $A u+A u$ at various energies.

Let me reemphasize that since our basic philosophy involves, in the first instance, using the on-shell hadron-hadron cross-sections as input to the multibody cascade, we have in principle no parameters; the two body inputs, baryon-baryon, meson-baryon and meson-meson are obtained from already performed experiments. Of course in practice there are gaps in this matrix of cross-sections and more seriously we have no direct knowledge of resonant-resonant interactions. We in fact make the minimal assumption that B-B, B-M, M-M interactions are essentially identical to $\mathrm{N}-\mathrm{N}, \mathrm{N}-\pi, \pi-\pi$.

\section{Au + Au AT THE AGS}

The question which immediately arises is: Can we indeed detect and measure high baryon densities, should they occur in Au+Au collisions? In Fig. 1, we display ARC predictions, obtained in advance of the Au measurements, against the actual experimental data. We are grateful to M.C. $\operatorname{nin}^{3}$ for his participation in this analysis. It is at the same time both striking and somewhat depressing that the agreement of data with ARC predictions is so precise. However, Fig. 3 shows an artificial attempt to increase baryon transparency in the collision and thus to change the observed peak proton level. The differences, at the central rapidities not yet measured, due to the increased tranparency produced by less energy loss in $\mathrm{N}-\mathrm{N}$ collisions are, we believe, really observable. Hence 
E866 is truly capable of "seeing" high baryon density with heavy ions. This is not a trivial conclusion since there is some doubt that any existing data are sensitive to the hadronic equation of state. The present agreement at less central proton rapidities in Fig. 1 already suggests appreciable densities are achieved at AGS energy.

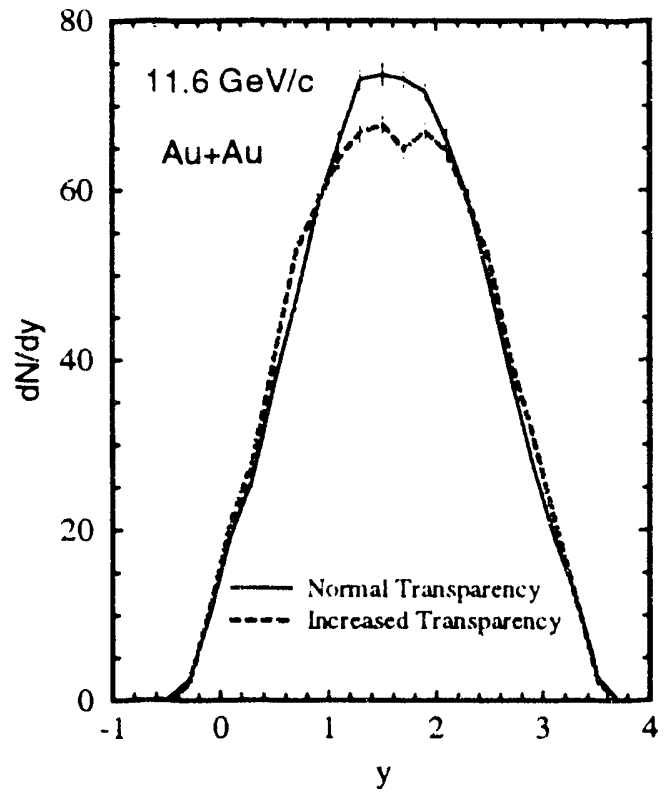

Figure 3. Proton rapidity spectra for increased tranparency in pp interactions leading to an observable reduction in the mid-rapidity peak.

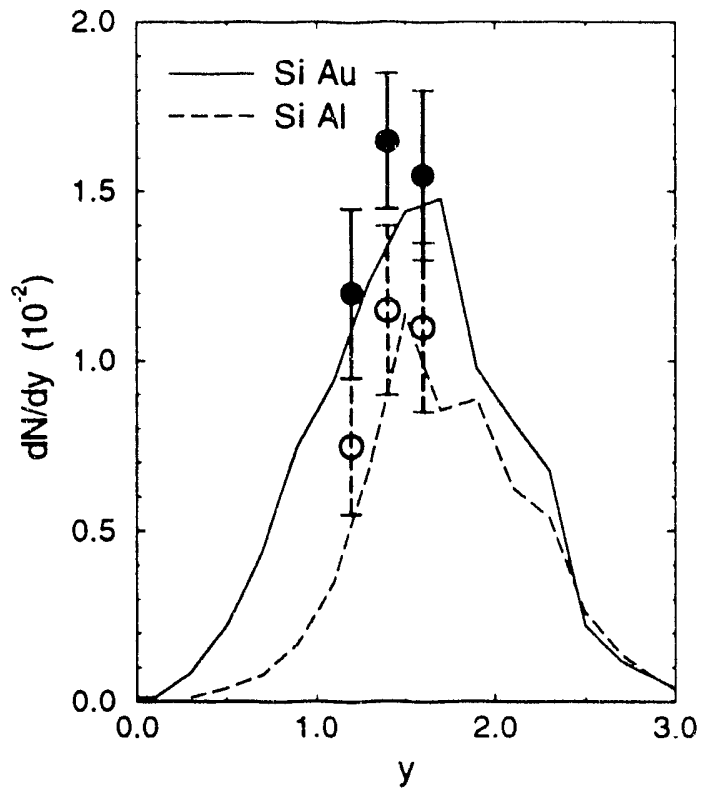

Figure 4. Antiproton production compared to E802. For SiAu screening increases yield by more than a factor of two.

At Borlange I showed snap shots of baryon density in the $A u+A u$ collisions as function of time, including one treating the target (or projectile) nucleons separately. By "colouring" target and projectile baryons differently one is able to gain valuable insight into the degree of stopping achieved in the collision and therefore to understand better the apparent enhancement in density. We can conclude in fact from Fig. 2 that although the local densities rise to more than 8 times normal nuclear densities, the relative motion between target and projectile baryons is so large that the operative densities are perhaps two-thirds of what they seem, i.e. the nuclei are still quite transparent. Nevertheless, truly high nuclear densities are achieved and in the presence of considerable stopping. Somewhat higher densities can be anticipated at beam momenta lower than the present $11.6 \mathrm{GeV} / \mathrm{c}$, and the largest deviations from theoretical spectra may well appear below the energy for maximum density. An ad hoc equation of state can be inserted into the cascade to simulate high density effects. The simplest to accommodate is just the effect of exclusion at the quark level and the best observable to focus on is probably $\mathrm{K}^{+}$ production. At $11.6 \mathrm{GeV} / \mathrm{c}$ preliminary estimates of increased $K^{+}$are small.

\section{ANTIPROTON PRODUCTION}

An argument similar to that presented for strangeness suggests that anomalously high levels of anti-nucleons are also possible for ion collisions in which quark-gluon plasma is formed. Pertinent data exists at the AGS and previous theoretical analysis ${ }^{5}$ has had considerable difficulty in obtaining sufficient antiprotons. The problem is clear: AGS energies are only slightly above threshold for $\bar{p}$ production in $\mathrm{pp}$, while the annihilation 
cross-section is large for the resultant low energy $\vec{p}$ 's. Using $A R C^{6}$, a three-body screening mechanism has been identified which strongly inhibits the absorption of produced anti-nucleons. This mechanism heralds the appearance of a problem for all rescattering codes at high density. Interactions for a process with cross-section $\sigma$ are triggered by closest approach distances less than $r_{0}=(\sigma / \pi)^{1 / 2}$. Should $r_{0}$ be less than the average distance between medium particles, it will be impossible to ignore third bodies. The large $p \bar{p}$ annihilation cross-section at low relative energy precipitates this crisis for $p$ production at even modest nuclear densities. A straightforward fix is to introduce an annihilation time-delay deduced from the $\bar{N} N$ relative velocity and finite distance apart. During the delay other nearby particles, generally pions, may collide with the $N$ or it.s targeted nucleon and prevent annihilation. Annihilation is after all a causal process, taking place only when the nucleon and antinucleon overlap.

Thus a straight forward approach, using no artificial production enhancement ${ }^{5}$ from non-hadronic mechanisms, can explain this more exotic process. In Fig. 4 comparison with E802 is shown ${ }^{6}$, indicating the extent of agreement.

\section{COMMENTS}

The considerable success achieved so far with a relativistic cascade gives us confidence that higher energies can be described similarily. A major uncertainty in this treatment. of ion-collisions is our lack of knowledge of resonance-baryon and resonance-resonance interations. The redundancy of data achievable at RHIC from wide control over both accelerating species, and energy, should help to throw light onto otherwise unknown regions. A great advantage of a hadronic cascade (even after including parton substructure) over more qualitative approaches, is its ability to "normalise" predictions. Should the QCD plasma prove easy to find experimentaliy, for example if large enhancements occur in all hadron $m_{t}$ spectra, such quantitative prediction might be a luxury. It is far more likely one will require a good quantitative estimate of what to expect in the absence of the plasma.

\section{REFERENCES}

1. S. H. Kahana, Y. Pang and T. J. Schlagel, HIPAGS'93, MIT, January 1993; Y. Pang, T. J. Schlagel and S. H. Kahana, Nucl. Phys. A544, 435c (1992).

2. Y. Pang, T. J. Schlagel and S. H. Kahana, Phys. Rev. Lett. 68, 2743 (1992); T. J. Schlagel, Y. Pang and S. H. Kahana, Phys. Rev. Lett. $\underline{69}, 3290$ (1992).

3. M. Gonin (E802/E866 Collaboration), BNL-47925.

4. E802 Collaboration, T. Abbott et al Phys. Rev. Lett. 64, 847 (1990); 66, 1567 (1991); E814 Collaboration, J. Barrett et al Phys. Rev. Lett. 64, 1219 (1990); P. Braun-Munzinger et al, Nucl. Phys. A. 544, 137c (1992), Proceedings of Quark Matter'91; S. A. Eiseman, et al, E810 Collaboration, BNL-47870, submitted to Physics Letters B.

5. J. Costales, Ph.D. Thesis, MIT (1990); A. O. Vaisenberg, et al, Eksp. Teor. Fiz. 29, 719, (1979); JETP Lett. 29, 661 (1979); A. Jahns, H. Stöcker, W. Greiner and H. Sorge, Phys. Rev. Lett. 68, 2895 (1992).

6. S. H. Kahana, Y. Pang, T. J. Schlagel and C. Dover, Phys. Rev. C 47, R1356 (1993). 

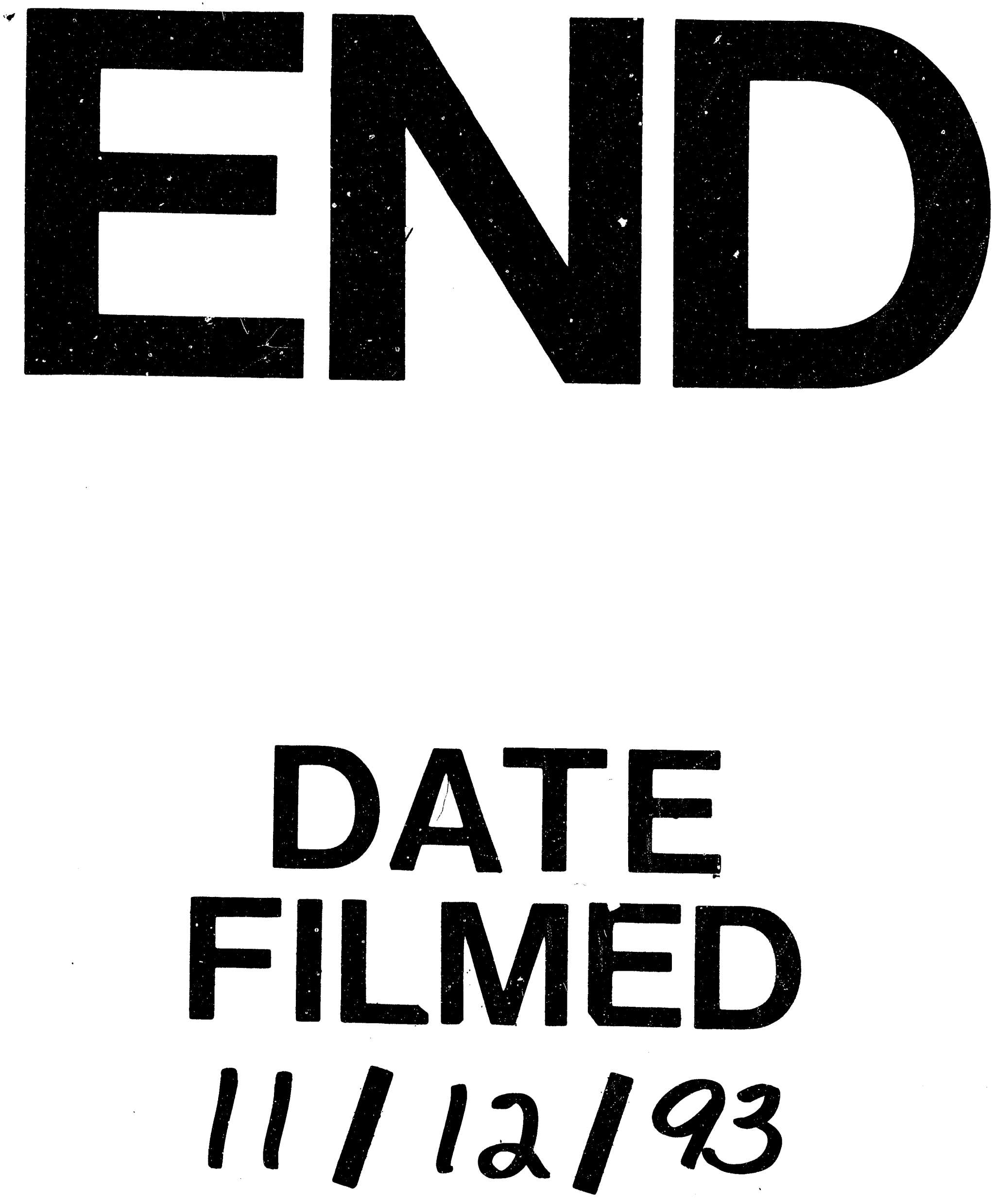
